\title{
Single Healthcare Portal - A Game Changer for the Healthcare Industry
}

\author{
Parag Shah* \\ Department of Statistics, H L College of Commerce, India \\ Received: 非 July 24, 2018; Published: 䟧 August 01, 2018 \\ *Corresponding author: Parag Shah, Department of Statistics, H L College of Commerce, India
}

\section{Mini Review}

We have observed a paradigm shift in healthcare information management over the past few years, activated by the birth of electronic medical records and medical informatics. This new world of electronically entered, stored, and exchanged medical information has addressed many of the shortfalls of the world of folders and paper medical records. This shift in information management is ongoing, with a focus now on the new focus on accountable, affordable, high quality and patient-centric healthcare. These include massive/large scale adoption of electronic health records by governments, hospitals and physicians to full digitization of patient records. If there is any single factor which unifies all these healthcare trends, it is Big Data - the huge gaps faced by the industry in converting unstructured information into meaningful business intelligence. Global healthcare expenditures are projected to reach a total $\$ 8.7$ trillion by 2020 , increased from $\$ 7$ trillion in 2015 , representing a CAGR of $4.3 \%$ during the five-year period.

Our understanding of a disease and its treatment is never complete. It's always a learning process. EHR (Electronic health record) of patients help doctors, health care providers and researcher to understand disease process better and help its treatment. We have been fighting cancer for more than 50 years. Our treatment has improved but we are for from understanding the disease. In fact, it is considered to be not one but many diseases. How it develops and how it mutates and defies treatment is not fully understood. We need all the data from all sources including, doctors, researchers and hospital for better understanding and health portal or EHR will able to provide these resources.

India has highest number of tuberculosis and death related to TB. Due to pathetic health care system and poor patients' database, the number of TB patients differ by hundreds of thousands. many thousands go unrecorded. We need to improve patient data of diseases like TB, Malaria, HIV/AIDS, maternal and infant death and recent epidemic of drug abuse as national priority. All these can be possible though health care portal such as EHR (electronic health records).

This article aims to showcase capabilities and advantages of the common platform for healthcare players across all stages of the data lifecycle. One uniform healthcare portal that integrates data from all sources to address the entire spectrum of healthcare. A Healthcare portal which allows to enter, store and share patient's data from different sources like doctor, medical laboratory, hospital, pharmacies and insurance (Figure 1).

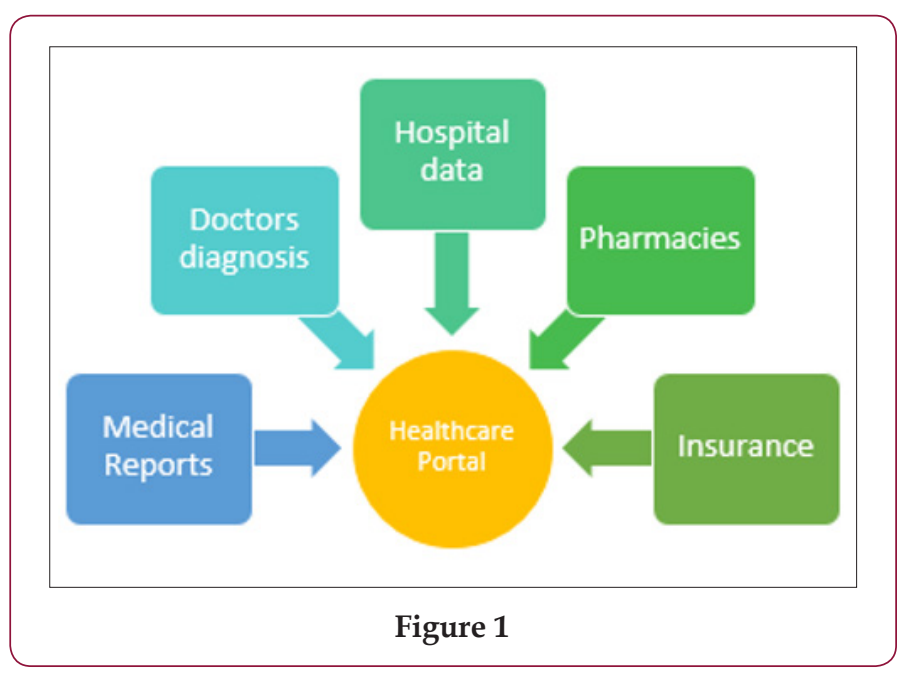

Through this Healthcare portal, a patient can share his medical reports with doctors or doctor's diagnosis with Hospitals or Hospital expenses with the Insurance company and more. This portal stores/will store complete history of the patient, which helps/will help the doctors to diagnose effectively. As you know, USA is almost fully EHR. Every doctors are using one or other electronic health records. It is all private and not controlled by government. Unfortunately, there are lots of EHR venders and everybody using different EHR and not connected to each other. Therefore, health care data are not shared and so optimal utilization of data in benefit of patients is not done.

In India, as you have mentioned in this article that if proposed health portal run through Government and if its only ONE system and all patients (general population) in this system link via one unique identifier (i.e. Aadhar Number) than anyone (doctors, hospital, researcher, laboratory, pharmacy, insurance) can access from anywhere anytime. There are many players which provide such services, and which are customized as per customers' needs. 
But, there is a dire need for a uniform portal across the industry which is implemented at all stages of data lifecycle and for all medical data sources. This can be done only if government takes up an initiative to develop such portal by taking inputs from all stake holders of healthcare industry. Once developed, every stake holder of this domain should be abided by law to use this Healthcare portal. Usage of such portal will solve many problems which the industry is facing like different database or different data format and data transfer. Moreover, data analytics plug-ins to this portal will be icing on the cake. This portal can help the healthcare industry to:

\section{Data Driven Decision by Clinician}

Making decisions based on instinct alone has never been sufficient. This is certainly true in healthcare. Clinical practitioners require data like medical reports, current medicines etc. to make their medical diagnosis, treatment recommendation, and prognosis. A richer set of near-real-time information can greatly help physicians determine the best course of action for their patients, discover new treatment options, and potentially save lives.

\section{Improved Coordination in Hospital System}

Even within the same hospital systems, it is very disorganized. This portal can be used to help patient with both within the systems and between/among all types of healthcare organizations across the country. Normally, there is lack of communication even within different specialties as well as among colleagues about a patient, and this portal can work to have all records reachable across the country. It also offers a number of potential benefits to providers, including administrative efficiencies, improved responsiveness to patients' needs, more effective care, and cost savings.

\section{Reduction of Frauds}

A significant amount of money has been lost in the healthcare industry due to fraud, it's important for insurance companies

ISSN: 2574-1241

DOI: $10.26717 /$ BJSTR.2018.07.001515

Parag Shah. Biomed J Sci \& Tech Res

(C) This work is licensed under Creative

Submission Link: https://biomedres.us/submit-manuscript.php to use this portal for insight into patient's information and what physicians are doing for their patients. Portal can track fraudulent and incorrect payments, as well as the history of an individual patient.

\section{Enhanced Wellness}

This portal holds great potential if offered to patients in conjunction with their treatment program because they create new opportunities for improving health care delivery and follow-up of clinical care. It can help healthcare organizations remind patients of medicines, check-up, follow-ups and to keep up with a healthy lifestyle, as well as keep track of where a patient stands in regard to their lifestyle choices.

\section{Rich Database for Research}

The major problem for healthcare research is unavailability of the structured data. With complete medical data of huge number of patients, it can also be a useful data resource for researchers.

\section{Policy decision by Government}

With the portal under government's control, the data secrecy issue can be resolved. Also, these data will serve as a good resource and a back bone for Government for taking policy decisions in the public health sector.

In spite of the potential advantages, the acceptance of such Healthcare portal may be low. The success depends on the degree of acceptance by its users, where health care professionals are key stakeholders to adoption and use. A number of barriers to acceptance have been identified, such as concerns about costs, added workload and workflow demands, technology literacy, liability issues, and confidentiality and privacy risks.

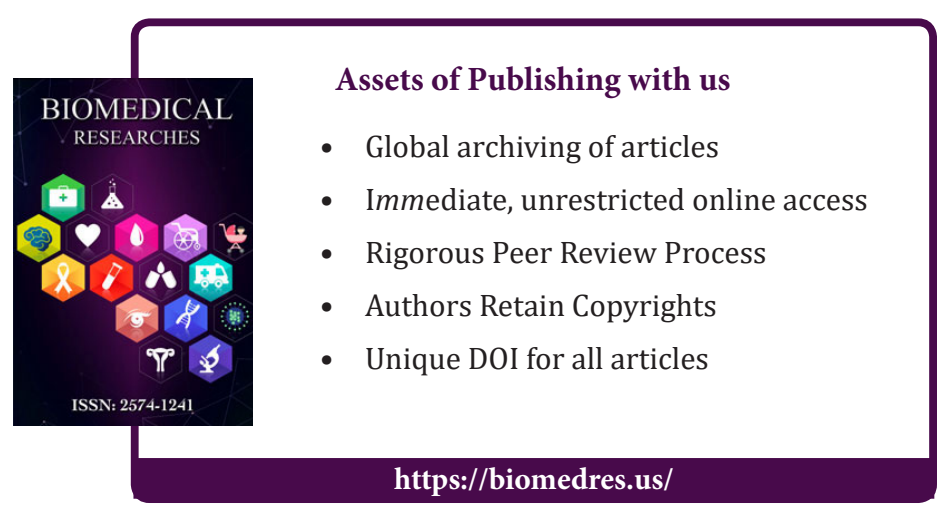

